OPEN ACCESS

Edited by:

Joško Osredkar,

University Medical Centre Ljubljana,

Slovenia

Reviewed by:

Bhakta Prasad Gaire,

University of Maryland, Baltimore,

United States

Federica Pessina

University of Siena, Italy

*Correspondence:

Xuan Zhao

zhaoxuan0323@hotmail.com

${ }^{\dagger}$ These authors have contributed

equally to this work

Specialty section:

This article was submitted to

Cellular Biochemistry,

a section of the journal

Frontiers in Cell and Developmental

Biology

Received: 03 December 2020 Accepted: 09 April 2021

Published: 08 June 2021

Citation:

Zhang T, Wang Y, Xia Q, Tu Z, Sun J, Jing $Q$, Chen $P$ and Zhao $X$ (2021) Propofol Mediated Protection of the Brain From Ischemia/Reperfusion Injury Through the Regulation of Microglial Connexin

43. Front. Cell Dev. Biol. 9:637233. doi: 10.3389/fcell.2021.637233

\section{Propofol Mediated Protection of the Brain From Ischemia/Reperfusion Injury Through the Regulation of Microglial Connexin 43}

\author{
Tingting Zhang ${ }^{\dagger}$, Yanyan Wang ${ }^{\dagger}$, Qin Xia ${ }^{\dagger}$, Zhiyi Tu, Jiajun Sun, Qi Jing, Pei Chen and \\ Xuan Zhao*
}

Department of Anesthesiology, Shanghai Tenth People's Hospital, Tongji University School of Medicine, Shanghai, China

Cerebral ischemia/reperfusion $(\mathrm{l} / \mathrm{R})$ injury is a serious condition that leads to increased apoptosis of microglial and neurons in the brain. In this study, we identified that Cx43 expression level is significantly increased in the microglial cells during $\mathrm{I} / \mathrm{R}$ injury. Using an in vitro model (hypoxia/reoxygenation-H/R injury), we observed that $\mathrm{H} / \mathrm{R}$ injury leads to an increase in activation of microglial cells and increase in levels of pro-inflammatory markers such as IL-1 $\beta, I L-6$, and TNF- $\alpha$. Additionally, we could also observe significant increase in phosphorylation of $\mathrm{Cx} 43$ and Cav3.2 levels. To assess the role of $H / R$ injured microglial cells on neuronal population, we cultured the neurons with conditioned media (MCS) from H/R injured microglial cells. Interestingly, we observed that microglial $H / R$ injury significantly decreased Map2 expression and affected neuronal morphology. Further, we aimed to assess the effects of propofol on cerebral $H / R$ injury, and observed that $40 \mu \mathrm{M}$ propofol significantly decreased Cx43, Cx43 phosphorylation, and CaV3.2 levels. Additionally, propofol decreased apoptosis and increased Map2 expression levels in $\mathrm{H} / \mathrm{R}$ injured neurons. Using silencing experiments, we confirmed that siCx43 could significantly improve the propofol's rescue after $\mathrm{H} / \mathrm{R}$ injury in both microglia and neurons. We further developed an in vivo MCAO (middle cerebral artery occlusion) rat model to understand the effect of propofol in I/R injury. Interestingly, propofol treatment and downregulation of $\mathrm{C} \times 43$ significantly decreased the infract volume and apoptosis in these MCAO rats. Thus, this study clearly establishes that propofol protects the brain against I/R injury through the downregulation of $\mathrm{Cx} 43$ in microglial cells.

Keywords: Connexin 43, ischemia/reperfusion injury, microglia, propofol, apoptosis

\section{INTRODUCTION}

Ischemia/reperfusion injury (I/R injury) frequently develops during the recovery of blood supply after ischemia in the tissues (Kalogeris et al., 2012). During ischemia, tissues are deficient in oxygen and nutrients from the blood, and when circulation returns, the introduction of oxidative stress contributes to inflammation and oxidative damage (Kurian et al., 2016). Previous studies 
have shown that sometimes ischemia alone does not cause tissue damage, and that damage usually occurs when blood flow (reperfusion) is abruptly restored after a period of ischemia (Leithead et al., 2013; Jiang et al., 2014; Ponticelli, 2014). Reperfusion to restore blood supply to the brain for treatment of heart and cerebral ischemia might thus leading to brain damage. Situations affecting blood circulation such as surgery, organ transplantation, traumatic shock, thrombosis, and burns could lead to I/R injuries (Collard and Gelman, 2001). However, there are no adequate measures yet in place to prevent/protect organs against I/R injury. Additionally, the incidence of secondary brain I/R injury affecting mortality is high during perioperative time period (Mussa and Taggart, 2002; Ebrahimkhani et al., 2014). Hence, the focus of the anesthetics field has been to identify ways to avoid perioperative I/R cardiac and brain injury.

The major mechanisms involved in $\mathrm{I} / \mathrm{R}$ injury are excessive production of nitric oxide and other free radicals, inflammation, apoptosis, and homeostasis of calcium (Cheung, 2003). Microglia are immune cells which maintain the brain's homeostasis in the central nervous system (Taylor and Sansing, 2013). Studies have shown that I/R can activate microglia in the brain and alter its morphology (Yan et al., 2014; Surinkaew et al., 2018). Microglia in turn activates and secretes inflammatory factors (TNF $\alpha$, IL-1, IL-6, and PGE2) (Cai et al., 2014; He et al., 2016), which are related to neuronal apoptosis (Xia et al., 2015; Zhou et al., 2015). Microglia are neurotransmission regulators, and may affect astrocytes (Pascual et al., 2012). In previous study, a clear significant increase in $\mathrm{Ibal}^{+}$microglia and cell volume increases were observed in cerebral I/R injury brain (Chen et al., 2018). It is also important to understand that $\mathrm{I} / \mathrm{R}$ affects critical cerebral microcirculation exchange of substances and oxygen supply (Chen et al., 2018).

Connexins are proteins that are widely distributed throughout the body and are critical to the functioning of the heart and brain. Gap junctions created by connexins are required to transmit electrical current between the heart and nerve cells or to coordinate cellular functions through the exchange of chemical signals and energy substrates (Rash et al., 1998; Iyyathurai et al., 2013; Alstrom et al., 2015). Studies have indicated that connexin expression is affected by age and gender (Song et al., 2011; Schulz et al., 2015), as well as pathophysiological changes such as ischemia, post-myocardial remodeling and hypertension (Schulz et al., 2015). Connexin $43(\mathrm{Cx} 43)$ has been identified to be expressed in both the heart and brain tissues and is specifically highly expressed in the astrocytes followed by microglial cells, in the brain tissue (Giaume and Theis, 2010). Inflammatory factors can induce upregulation of $\mathrm{Cx} 43$ expression in cerebral capillaries, while inhibition of $\mathrm{Cx} 43$ expression may reduce inflammation and promote repair after spinal cord injury (Cronin et al., 2008). Additionally, mimic peptides that inhibit $\mathrm{Cx} 43$ activity can minimize vascular leakage and ganglion cell death following retinal ischemia (Danesh-Meyer et al., 2012). The expression of Cx43 is upregulated after CNS injury, and it is thought that Cx43 plays an important role in controlling the degree of this injury.
Cx43 plays a major role in $\mathrm{I} / \mathrm{R}$ injury in the heart and brain and has become a new focus for elucidating I/R injury (Schulz et al., 2015). Propofol and sodium thiopental anesthetics have been known to minimize the exchange of information between gap junctions and weaken the electrophysiological activities in cultivated hippocampal slices (Wentlandt et al., 2006). Propofol can also significantly inhibit the hemichannel activity of LPSinduced cortical astrocytes. Ketamine and dexmedetomidine does also have similar effects, but the inhibitory efficiency is only second to propofol (Liu et al., 2016). Many studies in the recent years have shown that anesthetics (propofol, isoflurane) can inhibit apoptosis, oxidative stress, inflammation and other protective effects on brain I/R (Ji et al., 2015; Tao et al., 2016; Yan et al., 2016).

In summary, cerebral $I / R$ injury affects the activation of microglial cells and induces neuronal apoptosis and additionally it influences the expression and activity of connexins such as Cx43. Further, as anesthetics such as propofol and ketamine have been identified to influence hemichannel activity of gap junctions formed by connexins. Further using in vitro and in vivo $\mathrm{I} / \mathrm{R}$ models, we have characterized the $\mathrm{I} / \mathrm{R}$ damaged microglia, neurons through assessment of Iba1, Cx43 and Cav3.2 levels. Additionally, we also assessed the role of propofol in cerebral I/R injury. Hence, this study clearly establishes the role of $\mathrm{Cx} 43$ in the pathogenesis of cerebral I/R injury and identifies the potential mechanism behind propofol's protection against I/R injury.

\section{MATERIALS AND METHODS}

\section{Materials}

Propofol (2,6-diisopropylphenol) was obtained from SigmaAldrich (United States). For in vivo experiments, propofol was used at a dose of $50 \mathrm{mg} / \mathrm{kg}$ and for in vitro experiments propofol from 5-80 $\mu \mathrm{M}$ concentrations were used. The antibodies used in this study were Anti-CACNA1H (Cav3.2, ab128251, and Abcam), Anti-Connexin 43/GJA1 (phospho S368, ab30559, Abcam), Anti-Connexin 43/GJA1 antibody (ab230537, Abcam), Anti-Ibal antibody (ab178846, Abcam), Gap27 (HY-P0139, MedChemExpress), and Map2 (ab11268, Abcam).

\section{Animals}

SD male rats $(300 \pm 10 \mathrm{~g})$ were purchased from Shanghai SLAC Laboratory Animal Co., Ltd. (Shanghai, $n=35$ in total). Animals were further housed in our animal facility with a $12 \mathrm{~h}$ light/dark cycle and $23 \pm 1^{\circ} \mathrm{C}$ temperature $(60 \pm 10 \%)$ humidity. Throughout the study, food and water were provided ad libitum for the animals. All experiments were performed with respect to NIH's principles of laboratory animal care. Further, all the experimental procedures were approved by the Institutional animal care use and ethics committee at the Tongji University School of Medicine.

\section{In vivo I/R MCAO Model}

Middle cerebral artery occlusion (MCAO) in SD rats ( $n=24)$ were generated using previously published protocol 
(Longa et al., 1989). Initially, rats were exposed to 5\% isoflurane (in $\mathrm{N} 2 \mathrm{O} / \mathrm{O} 2,7: 3$ ) to achieve anesthesia and to maintain the rats in anesthetized state $2 \%$ isoflurane was employed. The left common and bifurcated carotid artery were exposed through a midline incision in the neck as well as ICA and ECA were separated carefully. Occlusion was achieved by insertion of a silicon rubber-coated monofilament (360 $\pm 5 \mu \mathrm{m}$ in diameter) into the external carotid artery and advanced to ICA $18-20 \mathrm{~mm}$ from the carotid bifurcation. The monofilament was withdrawn after 90 min thus allowing reperfusion. Temperature of the rat was maintained at $37 \pm 0.5^{\circ} \mathrm{C}$ throughout the procedure and lidocaine ointment was applied to the surgical sites to decrease the post-operative pain and supplemented with normal saline when necessary which was similar to the previous study (Lee et al., 2020). Further, validation of the procedure was assessed using neurobehavioral analysis.

\section{Neurological Evaluation}

Neurological evaluation was performed $48 \mathrm{~h}$ after induction of ischemia and scores were assigned on a 6-point scale. For no neurological deficit-0, for a failure to extend left forepaw fully1 , for circling more toward the left- 2, for falling to the left3 , with no spontaneous movement and with decreased level of consciousness- 4 , and death-5.

\section{TTC Staining}

Brains were isolated $72 \mathrm{~h}$ after I/R and quickly frozen before sectioning into $2 \mathrm{~mm}$ coronal sections. Further, the sections were stained using 2\% TTC (sigma, United States) in saline solution for $30 \mathrm{~min}$ at $37^{\circ} \mathrm{C}$. Slices were further imaged and quantified using Image Pro Plus software. Further, the volume of infarction was calculated by multiplying the lesion area and the section thickness.

\section{Primary Cell Culture}

Primary microglial cells were obtained from neonatal SD rats as previously described (Wang and Zhou, 2018). Microglial cells were further cultured in DMEM medium containing 10\% FBS and $1 \% \mathrm{P} / \mathrm{S}$ at $37^{\circ} \mathrm{C}$ in $5 \% \mathrm{CO}_{2}$ till necessary. Primary rat neuronal cells were obtained from neonatal SD rats as described (Xu et al., 2012). The cells were cultured in neurobasal medium with $10 \% \mathrm{~B} 27$ and $1 \% \mathrm{P} / \mathrm{S}$.

\section{$H / R$ in vitro Model}

$\mathrm{H} / \mathrm{R}$ injury in the microglial cell model were achieved by culturing the cells in a glucose free culture medium under hypoxic conditions at $37^{\circ} \mathrm{C}$ with $5 \% \mathrm{CO}_{2}$ and $1 \% \mathrm{O}_{2}$ for $2-24 \mathrm{~h}$. Further, reoxygenation of cells were performed with $5 \% \mathrm{CO}_{2}$ and $21 \% \mathrm{O}_{2}$ for $12 \mathrm{~h}$ in full culture medium. Based on the results from MTT assay, we choose $12 \mathrm{~h}$ as the hypoxia time point. All subsequent experiments were carried out with $12 \mathrm{~h}$ of hypoxia and $12 \mathrm{~h}$ of reoxygenation $\left(\mathrm{H}_{12} / \mathrm{R}_{12}\right)$. Microglial cells underwent $\mathrm{H} / \mathrm{R}$ injury and after $24 \mathrm{~h}$ (H12/R12), microglial cell supernatant (MCS) were collected and centrifuged at 1,500 rpm for $5 \mathrm{~min}$. Further, neuronal cells were cultured with the MCS medium for $24 \mathrm{~h}$ to assess the role of H/R injury onto the neuronal cells.

\section{MTT Assay}

On 96 well plate, cells were initially seeded. After H/R injury, wells were cultured with Methylthiazolyldiphenyl-tetrazolium bromide (MTT) $(0.5 \mathrm{mg} / \mathrm{ml}$, Sigma) in a humidified incubator at $37^{\circ} \mathrm{C}(5 \% \mathrm{CO} 2)$ for $90 \mathrm{~min}$. Further, cells were treated with $100 \mu \mathrm{l} /$ well of DMSO for $30 \mathrm{~min}$ in dark. The supernatant was transferred to a flat-bottom 96-well microtiter plate and measured at $560 \mathrm{~nm}$ using a spectrometer.

\section{ELISA Assay}

To assess IL-1 $\beta$, IL-6, TNF- $\alpha$, and IL-10 levels, we used ELISA assays obtained from Shanghai Jianglai Bio., and was performed according to the manufacturer's instructions. The absorbance was detected using a plate reader at the absorption of $405 \mathrm{~nm}$.

\section{Immunofluorescence}

The cells or were fixed using $4 \%$ paraformaldehyde at room temperature (RT) for $15 \mathrm{~min}$. The cells were then washed thoroughly with PBS and permeabilized with $0.25 \%$ triton$\mathrm{x}$ in PBS for $10 \mathrm{~min}$. Cells were blocked with 1\% BSA in PBST (PBS with $0.1 \%$ Tween-20) for $30 \mathrm{~min}$. Further, the cells were incubated with CACNA1H, Connexin 43, Map2 and Ibal antibody primary antibodies overnight at $4^{\circ} \mathrm{C}$. The cells were thoroughly washed with PBST and incubated with corresponding secondary antibodies for $1 \mathrm{~h}$ at RT. Again the cells were washed with PBST and stained with DAPI for 5 min. Finally, the cells were visualized and imaged using a fluorescence microscope (Leica, Germany). Immunofluorescence staining was also performed in the hippocampus and its adjacent tissues.

\section{TUNEL}

Cells were seeded onto dishes and allowed to attach for $12 \mathrm{~h}$ and undergo $\mathrm{H} / \mathrm{R}$, the TUNEL staining was carried out according to the manufacturer's instructions (Roche). Finally, the cells were visualized and imaged using a fluorescence microscope (Leica, Germany).

\section{Western Blotting}

Protein extraction was carried out using whole protein extraction kit (Solarbio, China). Further, protein concentration was assessed using Bradford assay. A total $20 \mu \mathrm{g}$ of the protein were loaded onto SDS-PAGE gel (4-15\%) and allowed to migrate for $1 \mathrm{~h}$. Further, the gel was transferred onto a nitrocellulose membrane and blocked using 5\% skim milk for $1 \mathrm{~h}$. Next, the membrane was incubated with respective primary antibodies overnight at $4^{\circ} \mathrm{C}$. The membranes were thoroughly washed with PBS-T and then incubated with corresponding secondary antibodies for $1 \mathrm{~h}$ at RT. The membranes were again washed and then incubated with chemiluminescence substrate (Thermo Scientific ${ }^{\mathrm{TM}}$ ) for $10 \mathrm{~min}$ followed by being imaged using a reader 60 .

\section{Statistical Analysis}

Data were assessed with Student's $t$-test and with One way Analysis of Variance (ANOVA) in three and more groups using 

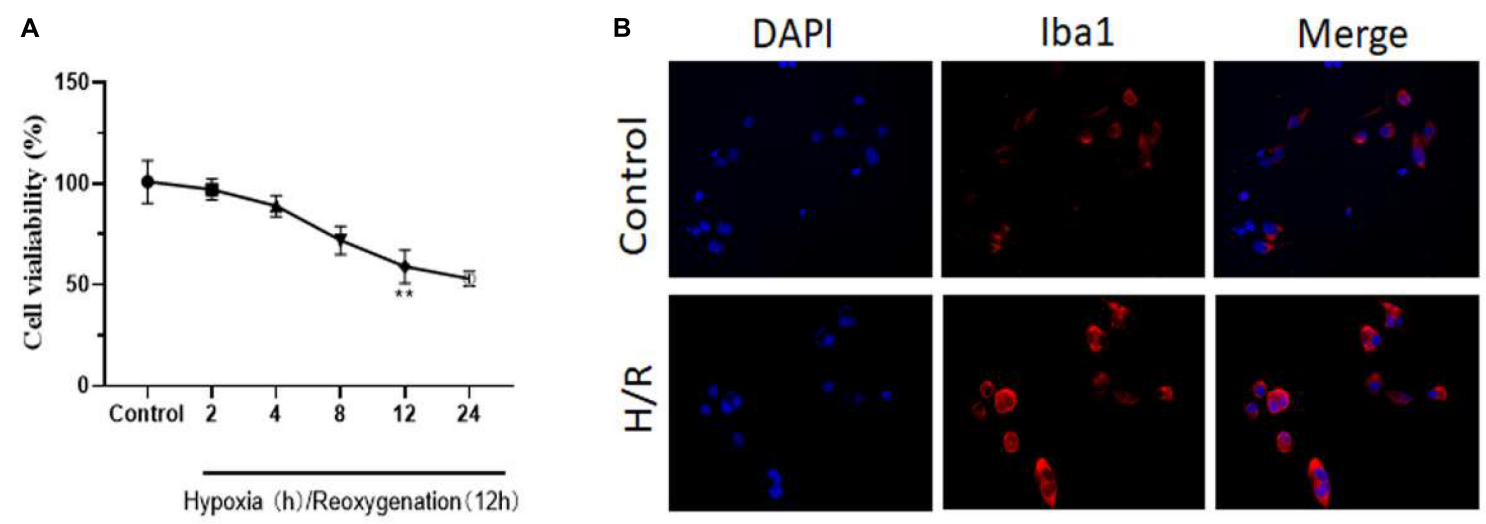

C
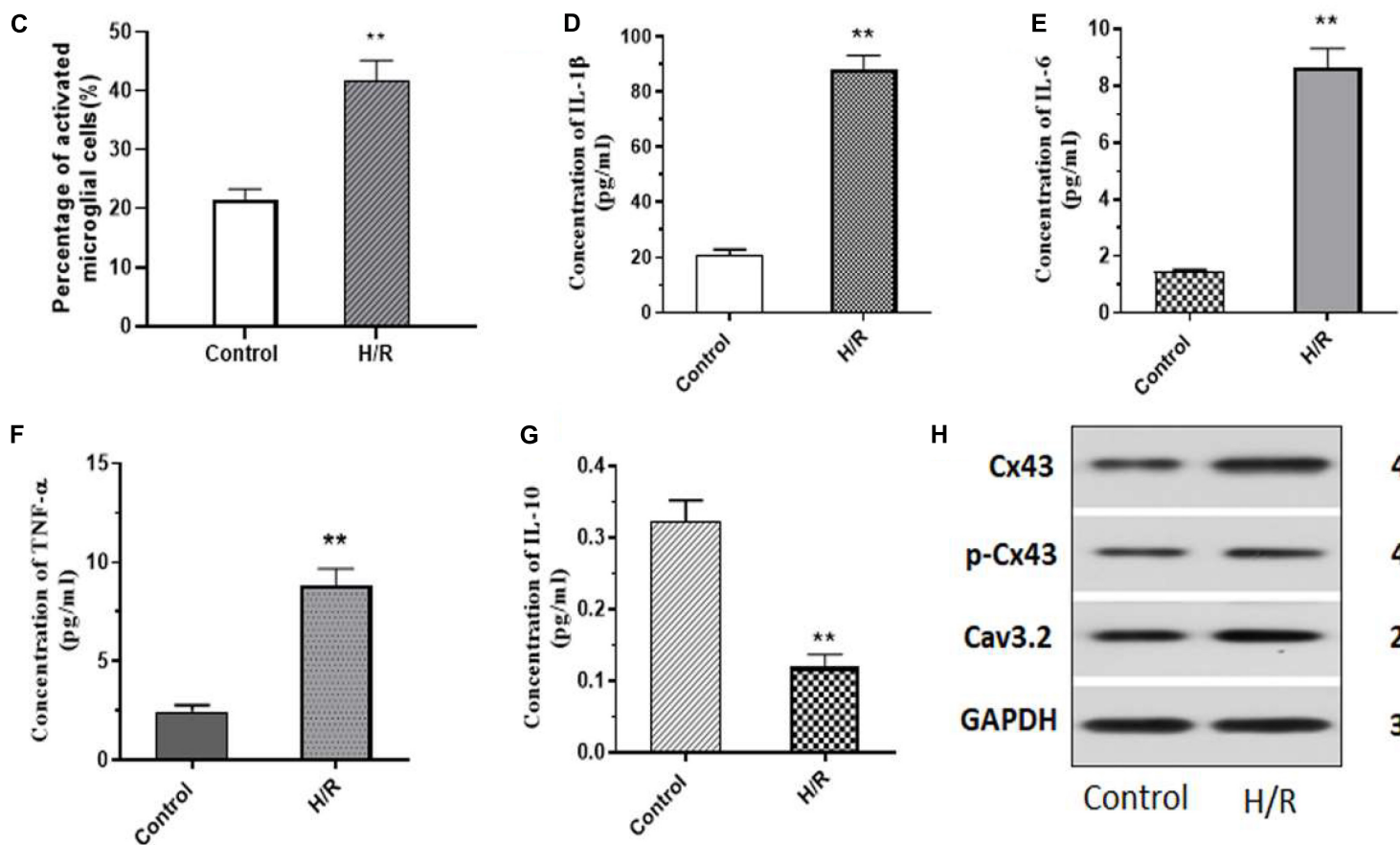

$43 K d$

$43 K d$

259Kd

37Kd

FIGURE 1| H/R induces microglia activation with an increase in Cx43 phosphorylation and Cav3.2 expression. (A) Cell viability as measured using MTT assay. (B) H/R induced activation of microglia with increasing Iba1 expression. (C) Number of Iba1+ cells (activated microglia) as assessed using ImageJ. (D-G) The levels of inflammatory cytokines of IL-1 $\beta, \mathrm{IL}-6, \mathrm{TNF}-\alpha$, and IL-10 were analyzed using ELISA. (H) Protein expression of Cx43, phosphorylation Cx43 and Cav3.2 as observed through western blotting. ${ }^{*} p<0.05,{ }^{* *} p<0.01$ vs. Control. $n=3$.

GraphPad Prism. All the data is presented as mean \pm the standard deviation (SD). Statistical significance is indicated on the figures as $* p<0.05,{ }^{* *} p<0.01$.

\section{RESULTS}

\section{H/R Induces Microglia Activation With Increasing Cx43 Phosphorylation and Cav3.2 Expression}

Initially, to assess the role of $H / R$ injury on cells, we isolated primary microglial cells from neonatal SD rats and induced H/R injury. All cells were treated in hypoxic conditions for 2-24 h, further the cells were reoxygenated for $12 \mathrm{~h}$. Evidentially, we observed a significant decrease in cell viability after 8-12 h of hypoxia which was further sustained till $24 \mathrm{~h}$ of hypoxia (Figure 1A). For subsequent experiments, all cells were treated with $12 \mathrm{~h}$ of hypoxia followed by $12 \mathrm{~h}$ of reoxygenation. Next, we performed some immunofluorescence (IF) staining on the cells after H/R injury and observed a significant $20 \%$ increase in $\mathrm{Iba1}^{+}$cells, indicating an increase in microglial activation after H/R injury (Figures 1B,C). Additionally, we analyzed the levels of inflammatory cytokines such as interleukin-1 $\beta$ (IL-1 $\beta$ ), IL-6, tumor necrosis factor (TNF- $\alpha$ ) and IL-10 after HR injury. We observed a significant increase in IL-1 $\beta$, IL-6, and TNF- $\alpha$ (Figures 1D-F). However, we observed a significant decrease in the levels of IL-10 (Figure 1G). Additionally, using western 


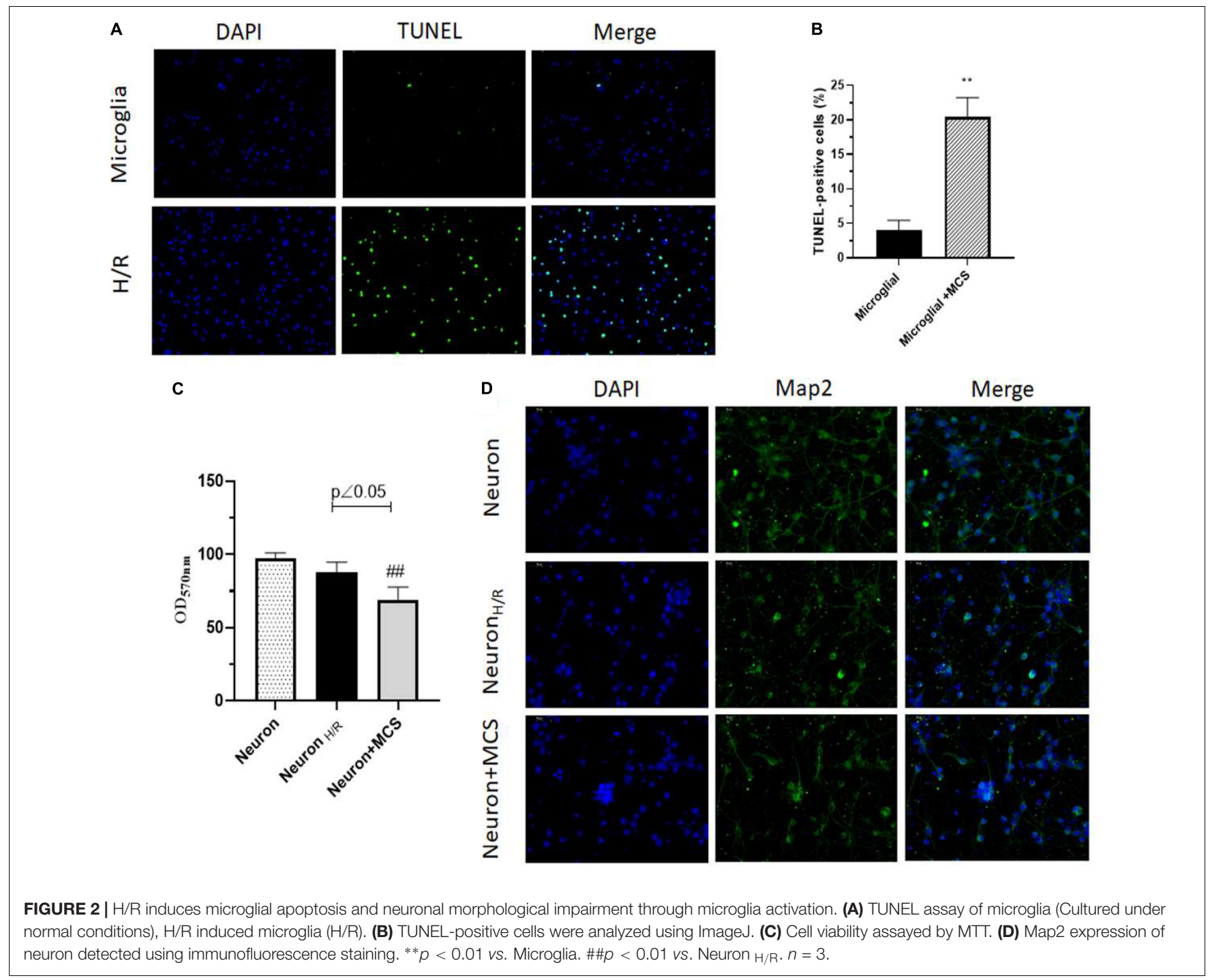

blotting we observed that post $H / R$, cells expressed increased levels of $\mathrm{Cx} 43$ and phosphorylated $\mathrm{Cx} 43$ (p-Cx43) (Figure 1H). Interestingly, we also observed a significant increase in the expression levels of T-type voltage gated calcium channel, Cav3.2, after exposure to H/R (Figure 1H).

\section{H/R Induces Microglial Apoptosis and Neuronal Morphological Impairment Through Microglia Activation}

To identify the role of H/R injury on cell apoptosis, we performed TUNEL assay. And, we observed that H/R significantly increased apoptosis in microglial cells (Figures 2A,B). Further, to understand the role of $\mathrm{H} / \mathrm{R}$ injury in neurons, we used the supernatant of $\mathrm{H} / \mathrm{R}$ injured microglial cells (MCS, microglial cell supernatant) and cultured the rat primary neurons for $24 \mathrm{~h}$. Interestingly, using MTT assay, we observed that the viability of neurons decreased slightly after H/R injury, but the viability decreased more significantly after culture with MCS (Figure 2C). Further, using immunofluorescence staining, we observed a clear decrease in MAP2 positive neurons along with a decrease in axonal structures post H/R injury and treatment with MCS (Figure 2D). These results indicated the negative deleterious effects of $\mathrm{H} / \mathrm{R}$ injured microglial cells on neural viability and morphology.

\section{Propofol Inhibited the H/R Induced Activation and Cx43 Expression in Microglia}

As previous studies have indicated a potential role for propofol on I/R injury (Tao et al., 2016), in our study, we aimed to explore the role of propofol in microglial activation mediated neuron apoptosis and $\mathrm{Cx} 43$ expression involved in. To achieve this, initially primary rat microglial cells were $H / R$ injured and then treated with propofol at varying concentrations from 0-80 $\mu \mathrm{M}$ for $24 \mathrm{~h}$. Using MTT assay, we observed that post treatment with propofol there was a slight increase in H/R injured cell's viability. However, with $40 \mu \mathrm{M}$ propofol treatment there was a significant increase in cell viability, when compared to the 


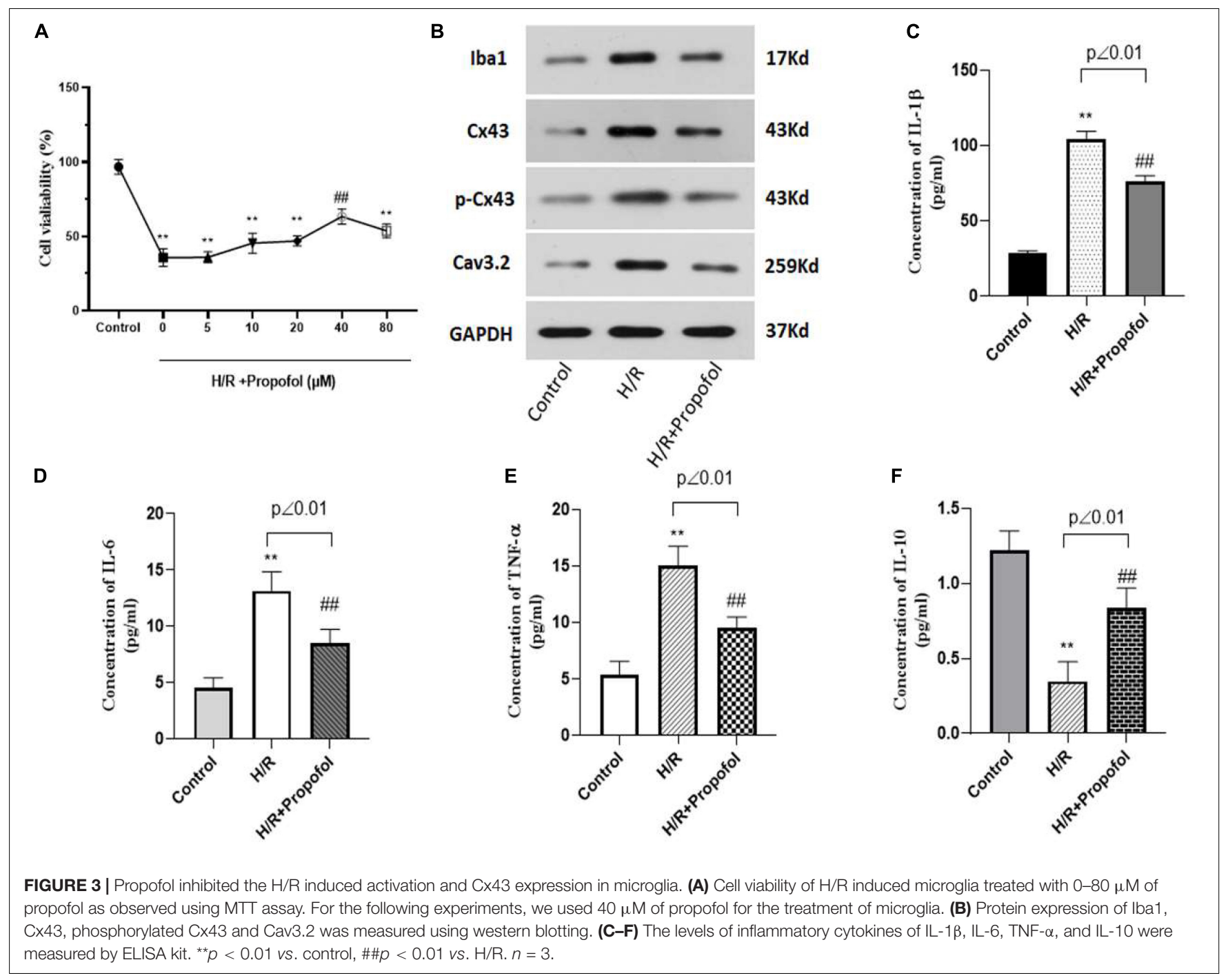

untreated H/R injured microglial cells (Figure 3A). Further, we also observed that $40 \mu \mathrm{M}$ propofol could significantly decrease Iba1, Cx43, Cx43 phosphorylation and Cav3.2 expression levels (Figure 3B). These results indicated that propofol could clearly decrease microglial activation and decrease phosphorylation and activation of $\mathrm{Cx} 43$. Additionally, we also observed a significant decrease in inflammatory cytokines such as IL-1 $\beta$, IL-6, and TNF- $\alpha$ (Figures 3C-E). However, we also observed a significant increase in the levels of IL-10 levels (Figure 3F). Hence, propofol treatment could significantly rescue the microglial cells from the deleterious effects of $\mathrm{H} / \mathrm{R}$ injury. Our results demonstrated that protects the brain against I/R injury through the downregulation of $\mathrm{Cx} 43$ in microglial cells to decreased neuron apoptosis.

\section{Knock Down of Cx43 Enhances the Effect of Propofol on Microglia Mediated Neuron Impairment Induced by H/R}

Based on the above-mentioned results, it was clear that activation of $\mathrm{Cx} 43$ plays a vital role in $\mathrm{H} / \mathrm{R}$ injury. Hence, we wanted to understand further if lack of $\mathrm{Cx} 43$ could affect propofol rescue on $H / R$ induced neuronal impairment. Similar to previous results propofol could rescue the Map2 expression and neuronal morphology in the cells treated with $H / R$ exposed MCS (Figure 4A). However, when the cells were silenced for Cx43 expression, there was a significantly higher rescue of Map2 expression and a higher morphological recovery among neurons (Figure 4A). Further, silencing of Cx43 and propofol treatment significantly rescued the cell viability of microglial cells, when compared to the H/R injured cells (Figure 4B). Additionally, silencing of $\mathrm{Cx} 43$ along with propofol treatment significantly decreased the inflammatory marker levels (IL$1 \beta$, IL-6, and TNF- $\alpha$ ) (Figures 4C-E). However, we also observed a significant increase in the levels of IL-10 levels (Figure 4F). Further, we assessed the levels of Iba1, Cx43, Cx43 phosphorylation and Cav3.2 levels using western blotting and based on these results it was clear that, silencing of $\mathrm{Cx} 43$ and propofol treatment could initially significantly decrease the expression of levels $\mathrm{Cx} 43$ and $\mathrm{Cx} 43$ phosphorylation (Figure 4G). Additionally, it could also significantly decrease Iba1 levels, 


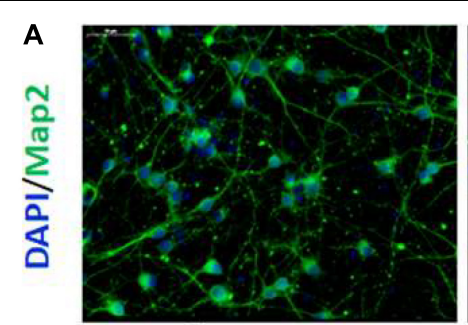

Neurons

B

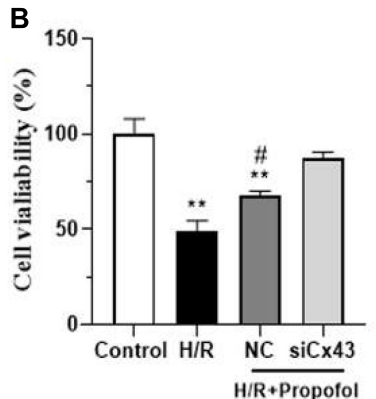

E

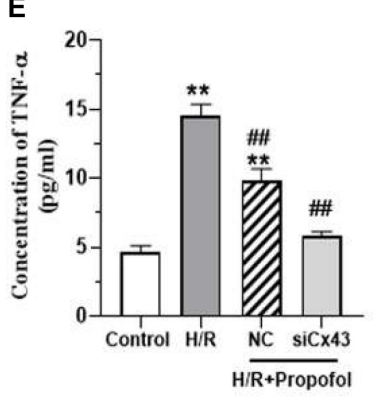

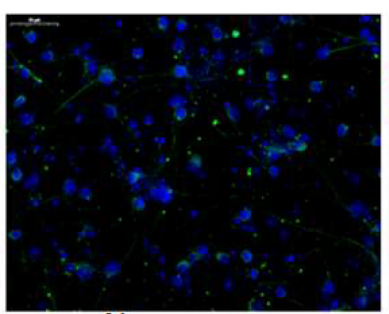

Neurons $+\mathrm{MCS}_{\mathrm{H} / \mathrm{R}}$

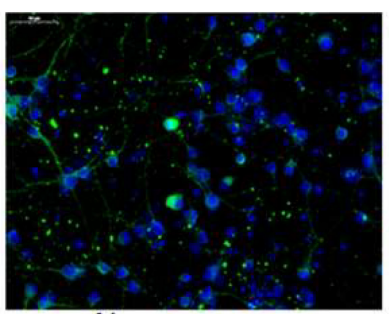

Neurons $+\mathrm{MCS}_{\mathrm{H} / \mathrm{R}+\mathrm{NC}+\text { Propofol }}$

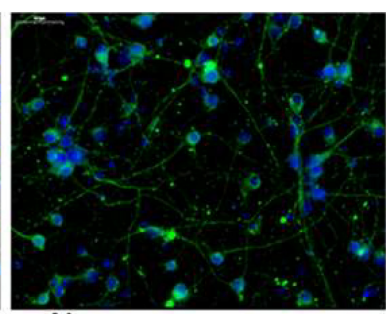

Neurons $+\mathrm{MCS}_{\mathrm{H} / \mathrm{R}+\mathrm{siC} \times 43+\text { Propofol }}$

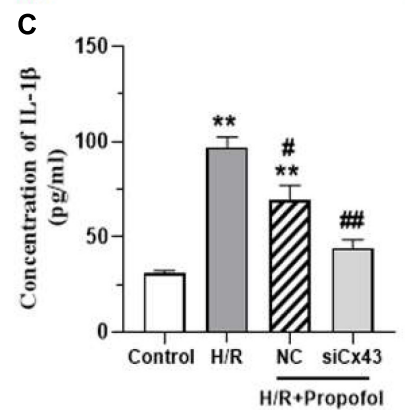

D

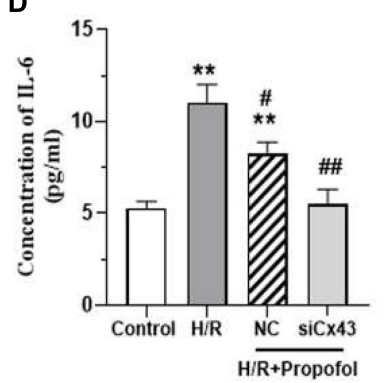

G

$\mathbf{F}$
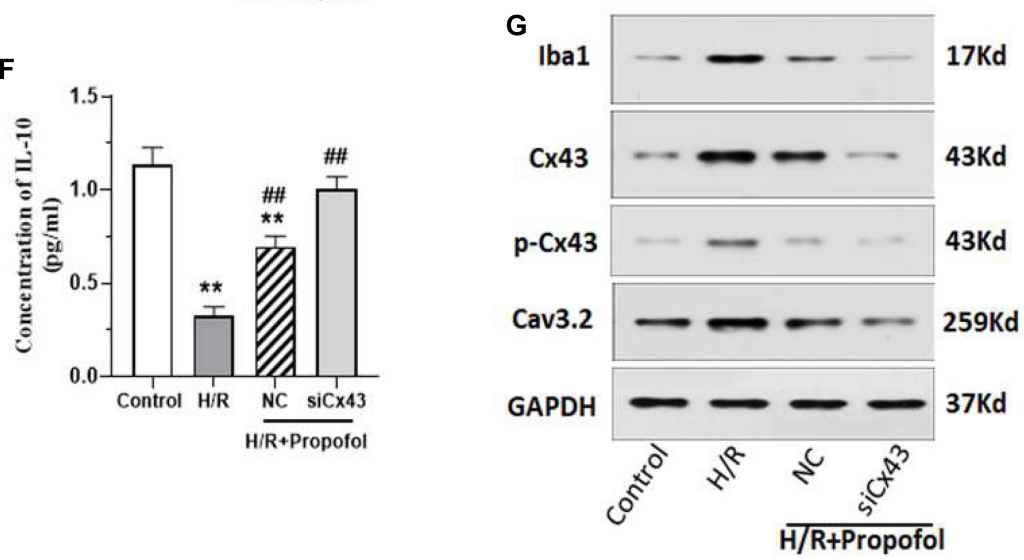

FIGURE 4 | Silencing of Cx43 expression enhances the effect of propofol on microglia mediated neuron impairment induced by H/R. (A) Expression of Map2 in neurons after treatment with/without microglial cell supernatant after H/R (MCS), treated with/without propofol and transfected with negative control (NC) or siRNA for $\mathrm{Cx43}$ (siCx43) was measured using immunofluorescence staining. (B) MTT cell viability assay of microglial cells which were w/wo H/R injury, transfected either with NC or siCx43 and treated with propofol. (C-F) The inflammatory cytokine IL-1 $\beta$, IL-6, TNF- $\alpha$, and IL-10 levels were measured using ELISA. (G) The protein expression of Iba1, Cx43, phosphorylation Cx43 (p-Cx43) and Cav3.2 was detected by western blotting. ${ }^{*} p<0.05$, ${ }^{* \star} p<0.01$ vs. Control. \#p $<0.05$, \#\#p $<0.01$ vs. H/R. $n=3$.

thereby indicating a decreased activation of microglial cells (Figure 4G). Similarly, we also observed a significant decrease in Cav3.2 levels. Interestingly, all these above-mentioned markers displayed levels almost similarly to the control uninjured cells. This indicated a potential complete rescue of the deleterious effects of $H / R$ injury with the aid of $\mathrm{Cx} 43$ silencing and propofol treatment.

\section{Propofol Alleviates H/R-Induced Neurological Impairment by Decreasing Cx43 and Cav3.2}

Further, we generated a rat middle cerebral artery occlusion (MCAO) model and checked the effect of propofol in rescuing induced injury. To confirm that propofol alleviates the H/R induced injury through the regulation of $\mathrm{Cx} 43$, we overexpressed $\mathrm{Cx} 43$ in the MCAO models. The rats were first separated into four groups: sham (untreated rats), MCAO (injured rats), MCAO treated with propofol and injected with negative control (empty plasmid) for Cx43 (MCAO + NC + propofol) and MCAO treated with propofol and intravenously injected with overexpression lentivirus for $\mathrm{Cx} 43$ (MCAO + $\mathrm{Cx} 43$ $\mathrm{OE}+$ propofol). We first assessed the neurobehavioral analysis score of these rats and observed that MCAO rats had a high neurological score, whereas treatment with propofol significantly decreased this score (Figure 5A). However, overexpression with $\mathrm{Cx} 43$ increased and worsened the behavior of these rats. Further, we visualized and measured the cerebral infract volume 

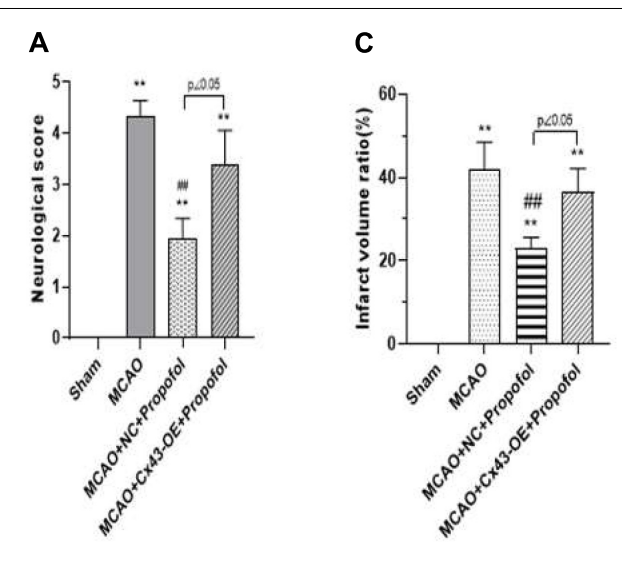

B

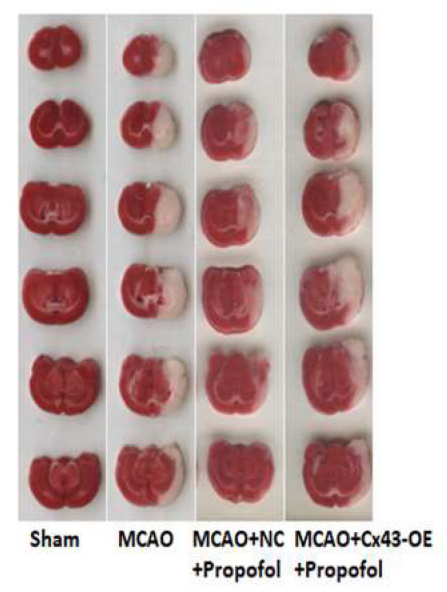

\section{D}
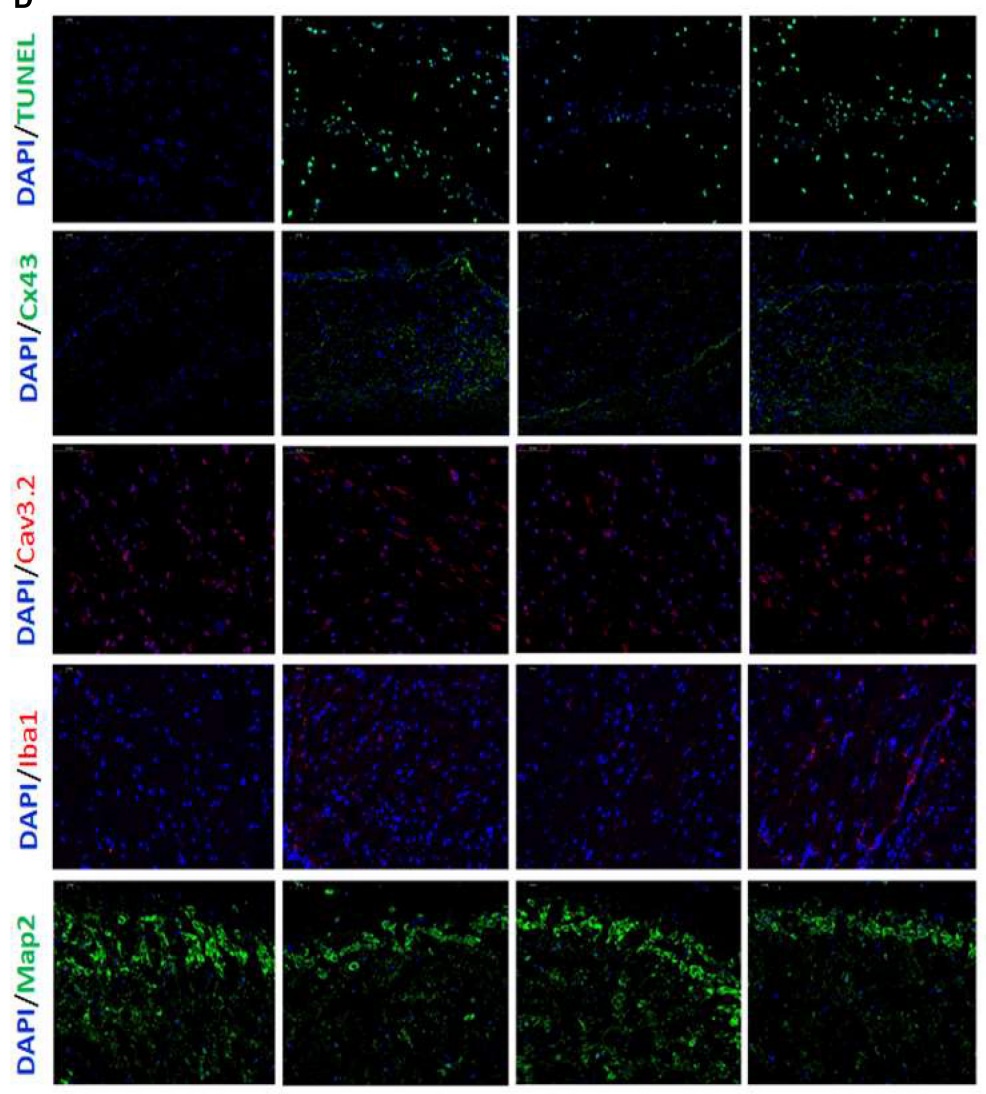

MCAO

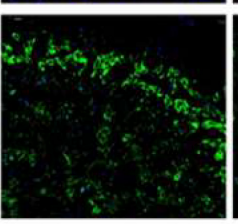

MCAO+NC

+Propofol

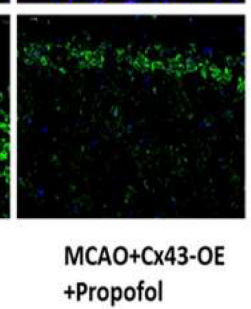

FIGURE 5 | Propofol alleviates H/R-induced neurological impairment by decreasing Cx43 and Cav3.2. Rat middle cerebral artery occlusion (MCAO) model was generated and animals were treated with/without propofol and intraventricularly injected with negative control (NC) or Cx43 overexpression lentivirus (Cx 43-OE). Sham group is used as the control here. (A) Neurobehavioral analysis is performed and scores are assessed. (B) TTC staining is used to assess the cerebral infarction, and the infarct volume was analyzed. (C) Apoptosis was measured using TUNEL staining and the protein expression and localization of Cx43, Cav3.2, Iba1, and Map2 was analyzed by immunofluorescence staining. ${ }^{\star} p<0.05,{ }^{\star \star} p<0.01$ vs. Sham. $\# p<0.05, \# \# p<0.01$ vs. MCAO. $n=3$.

using TTC staining and confirmed that the MCAO rats had a significantly higher injury/infract volume (Figure 5B). Further, we observed that the propofol treatment significantly decreased the injury or infract volume in these MCAO rats. However, $\mathrm{Cx} 43$ overexpression significantly decreased this recovery achieved by propofol. Further, we assessed vital markers such as TUNEL, Cx43, Cav3.2, Iba1, Map2 (Figure 5C). Initially, Cx43 staining confirmed that MCAO rats displayed high levels of $\mathrm{Cx} 43$ and propofol treatment clearly decreased Cx43 levels. Further, however, propofol treatment could not efficiently decrease the $\mathrm{Cx} 43$ levels in the $\mathrm{Cx} 43$ overexpression group. Further, TUNEL staining showed that the MCAO rats displayed increased levels of apoptosis, which could be evidentially decreased by propofol treatment. However, $\mathrm{Cx} 43$ overexpression, further increased the apoptosis levels despite the propofol treatment. Additionally, we could also observe an increase in number of cells expressing Iba1 and Cav3.2 in the MCAO rats compared to the sham rats. Propofol treatment could evidently decrease Ibal and Cav3.2 positive cells in the MCAO rats. However, Cx43 overexpression increased the levels of Iba1 and Cav3.2. Similar to our in vitro results, MCAO models also displayed decreased Map2 positive neuronal cells, however, propofol treatment could significantly recover this decrease. But, Cx43 overexpression did clearly worsen the Map2 expression levels even in the presence of propofol treatment. However, it is noteworthy that with all the above-mentioned evidence, it was clear that the even in the presence of $\mathrm{Cx} 43$ overexpression, propofol treatment could still decrease $\mathrm{Cx} 43$ levels and slightly recover the H/R associated injury. These results clearly indicate that propofol alleviates $\mathrm{H} / \mathrm{R}$ induced damage by decreasing $\mathrm{Cx} 43$ and Cav3.2 levels.

\section{DISCUSSION}

$\mathrm{I} / \mathrm{R}$ associated injury to the organs remain as the most prevalent inflammation associated damage to tissues and cells. In this study, we aimed to assess its role in brain microglial and we identified that $\mathrm{H} / \mathrm{R}$ injury induces the activation of the microglial cells. Further, it was clear that H/R injury increases microglial 
cell death, and release of pro-inflammatory markers such as IL-1 $\beta$, IL-6, TNF- $\alpha$. Additionally, we observed an increased expression of Ibal after exposure to H/R. Ibal is a prevalently used marker indicating the macrophage activation levels and clearly our results illustrated the status of the macrophages after H/R injury (Davis et al., 2017). Interestingly, we also observed that it significantly increased the levels of $\mathrm{Cx} 43$ (Figure 1). In the human system, glial cells encompasses $91 \%$ of cell populations in the central nervous system (Allen and Barres, 2009). Interactions between glia and neuronal cells are necessary for transmission of key information survival and functioning of the two populations (Giaume and Theis, 2010). This intercellular interaction is achieved primarily through gap junctions or through hemichannels. One such protein family are the connexins (cxs). Cxs as gap junctions allow cellcell interactions and exchanges of ions and small molecules, whereas as hemichannels they allow release of factors and other molecules into the external medium (Harris, 2007). Cx43 is majorly expressed in glial cells and vascular endothelial cells, and are identified to be primarily expressed after spinal injury (Naus et al., 1991; Danesh-Meyer et al., 2012). Importantly, it has been observed that upregulation of $\mathrm{Cx} 43$ immediately after ischemia may increase spreading of apoptotic signals, cellular debris and other dying cells to neighboring healthy environments thereby compounding the injury to the tissue and initiating new cell death signals (Frantseva et al., 2002). Cx43 upregulation has been observed in many ischemic diseases such as stroke, brain ischemia, brain and spinal cord injury (Hossain et al., 1994; Nakase et al., 2004; Ohsumi et al., 2006). Many recent studies have focused on the decrease of such gap junctions using small molecules or peptides post injury (Danesh-Meyer et al., 2008). However, it is essential to identify strategies which is employable either during pre-operative or peri-operative situations to minimize surgical associated I/R injury. Additionally, another key observation form our results are increased expression of CaV3.2 calcium channels after H/R injury, which has been associated with increased $\mathrm{Ca}^{2+}$ ion entry leading to mitochondrial overload and thus increased cell death under ischemic conditions (Gouriou et al., 2013).

Hence, from our evidence it was clear that microglial cells were highly affected by $H / R$ injury. However, we further wanted to assess the effect of $H / R$ injured microglial cells on neuronal cells. Interestingly, we observed that the MCS from H/R injured cells significantly decreased Map2 expression and increased apoptosis of neuronal cells (Figure 2). These results clearly indicated the effect of $H / R$ injury on neuronal cells through the release of factors by $H / R$ injured microglia cells. The effect of $\mathrm{Cx} 43$ hemichannels releasing microglial proinflammatory along with other paracrine and autocrine factors could significantly affect the morphology and physiological state of neuronal cells. These results clearly indicated for a need to develop treatment strategies associated with downregulation of hemichannel and gap junction associated proteins during I/R injury. Previously, studies have indicated the effect of anesthetics to inhibit gap junction and hemichannel activity in both astrocytes and neurons (Liu et al., 2016). Initially, we identified that propofol indeed does increase the cell viability and decrease $\mathrm{Cx} 43$ expression levels in H/R injured cell models. Additionally, we observed that propofol decreases Ibal levels in microglial cells thereby decreasing the activation of these cells after H/R injury (Figure 3).

Further, we were interested to understand the pathway through which propofol rescued cells from I/R injury. And interestingly, our results indicated that propofol rescued the cells from I/R injury through the downregulation of $\mathrm{Cx} 43$. Our results were further strengthened through silencing experiments, which showed that silencing of $\mathrm{Cx} 43$ significantly decreased negative effects of $\mathrm{H} / \mathrm{R}$ injury in both microglial cells and neurons (Figure 4). This evidence further strengthened our understanding of the interactions between neuronal and glial populations along with clarifying the roles of $\mathrm{Cx} 43$ in I/R injury. Finally, we could also prove the effect of propofol in rescuing from I/R injury using MCAO models. Evidentially, we could clearly establish that propofol could rescue the MCAO rats by decreasing the infract volume through the decrease of $\mathrm{Cx} 43$, Cav3.2, and Iba1 levels (Figure 5). Further, using overexpression models, we could establish that $\mathrm{Cx} 43-\mathrm{OE}$ worsened the injury associated with $\mathrm{I} / \mathrm{R}$ and propofol treatment could still slightly decrease the cell death and microglial activation through regulation of $\mathrm{Cx} 43$ expression. Interestingly previous studies have shown that propofol, an agonist of $\mathrm{GABA}_{\mathrm{A}}$ receptor and ketamine, an antagonist of glutamatergic channels could efficiently inhibit $\mathrm{Cx} 43$ based gap junction and hemichannel based communication. In this study, we further wanted to elucidate its role and understand its association with $\mathrm{Cx} 43$. Importantly, all the previous studies have been performed on hippocampal slices or organotypic cultures (Liu et al., 2016), this is the first study to show in an in vitro and in vivo MCAO based model, that indeed $\mathrm{H} / \mathrm{R}$ injury affects neuronal populations through activation of microglial $\mathrm{Cx} 43$ and indeed propofol could be used to improve the neuronal morphology and Map2 expression during H/R injury. Therefore, the current study provides insight into the potential use of propofol to treat or prevent cerebral I/R injury.

Our results indicated in Figure 1A, there was almost $40 \%$ cell death at $12 \mathrm{~h}$ after H/R as well as in Figure 2B only $20 \%$ cell death was observed. MTT assayed the viability of living cells (Figure 1A). Cell death includes active cell death-programmed death, apoptosis and passive cell death (cell necrosis). TUNELpositive cells are apoptosis cells (Figure 2B). Therefore, the apoptosis cells may be less to death cells. Moreover, the TUNELpositive cells were analyzed according to the photographs, among which there may be a difference. The effect of propofol on $\mathrm{H} / \mathrm{R}$ induced brain injury may be at least in part due to downregulation $\mathrm{Cx} 43$ phosphorylation levels in our study although propofol also shows effect in connexin silenced cells. We would perform further study to explore the exact act of propofol in $\mathrm{Cx} 43$ regulation during $\mathrm{H} / \mathrm{R}$ induced brain injury.

\section{DATA AVAILABILITY STATEMENT}

The data in this study can be obtained from the corresponding author. 


\section{ETHICS STATEMENT}

The animal study was reviewed and approved by Institutional animal care use and Ethics Committee at the Shanghai Tenth People's Hospital.

\section{AUTHOR CONTRIBUTIONS}

TZ, YW, and QX designed and completed experiments and completed the manuscript. TZ, ZT, and JS were responsible

\section{REFERENCES}

Allen, N. J., and Barres, B. A. (2009). Neuroscience: glia - more than just brain glue. Nature 457, 675-677. doi: 10.1038/457675a

Alstrom, J. S., Stroemlund, L. W., Nielsen, M. S., and Macaulay, N. (2015). Protein kinase $\mathrm{C}$-dependent regulation of connexin43 gap junctions and hemichannels. Biochem. Soc. Trans. 43, 519-523. doi: 10.1042/bst20150040

Cai, Z., Hussain, M. D., and Yan, L. J. (2014). Microglia, neuroinflammation, and beta-amyloid protein in Alzheimer's disease. Int. J. Neurosci. 124, 307-321. doi: 10.3109/00207454.2013.833510

Chen, Y., Wang, L., Zhang, Y., Zhou, Y., Wei, W., and Wan, Z. (2018). The effect of therapeutic mild hypothermia on brain microvascular endothelial cells during ischemia-reperfusion injury. Neurocrit. Care 28, 379-387. doi: 10.1007/s12028017-0486-4

Cheung, R. T. (2003). The utility of melatonin in reducing cerebral damage resulting from ischemia and reperfusion. J. Pineal. Res. 34, 153-160. doi: 10.1034/j.1600-079x.2003.00034.x

Collard, C. D., and Gelman, S. (2001). Pathophysiology, clinical manifestations, and prevention of ischemia-reperfusion injury. Anesthesiology 94, 1133-1138. doi: 10.1097/00000542-200106000-00030

Cronin, M., Anderson, P. N., Cook, J. E., Green, C. R., and Becker, D. L. (2008). Blocking connexin43 expression reduces inflammation and improves functional recovery after spinal cord injury. Mol. Cell. Neurosci. 39, 152-160. doi: 10.1016/j.mcn.2008.06.005

Danesh-Meyer, H. V., Huang, R., Nicholson, L. F., and Green, C. R. (2008). Connexin43 antisense oligodeoxynucleotide treatment down-regulates the inflammatory response in an in vitro interphase organotypic culture model of optic nerve ischaemia. J. Clin. Neurosci. 15, 1253-1263. doi: 10.1016/j.jocn. 2008.08.002

Danesh-Meyer, H. V., Kerr, N. M., Zhang, J., Eady, E. K., O'carroll, S. J., Nicholson, L. F., et al. (2012). Connexin43 mimetic peptide reduces vascular leak and retinal ganglion cell death following retinal ischaemia. Brain 135, 506-520. doi: 10.1093/brain/awr338

Davis, B. M., Salinas-Navarro, M., Cordeiro, M. F., Moons, L., and De Groef, L. (2017). Characterizing microglia activation: a spatial statistics approach to maximize information extraction. Sci. Rep. 7:1576.

Ebrahimkhani, M. R., Daneshmand, A., Mazumder, A., Allocca, M., Calvo, J. A., Abolhassani, N., et al. (2014). Aag-initiated base excision repair promotes ischemia reperfusion injury in liver, brain, and kidney. Proc. Natl. Acad. Sci. U. S. A. 111, E4878-E4886.

Frantseva, M. V., Kokarovtseva, L., and Perez Velazquez, J. L. (2002). Ischemiainduced brain damage depends on specific gap-junctional coupling. J. Cereb. Blood Flow Metab. 22, 453-462. doi: 10.1097/00004647-200204000-00009

Giaume, C., and Theis, M. (2010). Pharmacological and genetic approaches to study connexin-mediated channels in glial cells of the central nervous system. Brain Res. Rev. 63, 160-176. doi: 10.1016/j.brainresrev.2009.11.005

Gouriou, Y., Bijlenga, P., and Demaurex, N. (2013). Mitochondrial Ca2+ uptake from plasma membrane Cav3.2 protein channels contributes to ischemic toxicity in PC12 cells. J. Biol. Chem. 288, 12459-12468. doi: 10.1074/jbc.m112. 428128

Harris, A. L. (2007). Connexin channel permeability to cytoplasmic molecules. Prog. Biophys. Mol. Biol. 94, 120-143. doi: 10.1016/j.pbiomolbio.2007.03.011 for the isolation of primary cells. QJ and PC statistical analyzed the data. XZ directed the study and revisesed the manuscript. All authors contributed to the article and approved the submitted version.

\section{FUNDING}

This study was supported by the National Natural Science Foundation of China (No. 81873787). Natural Science Foundation of Shanghai (No. 18ZR1424800).

He, G. L., Luo, Z., Shen, T. T., Li, P., Yang, J., Luo, X., et al. (2016). Inhibition of STAT3- and MAPK-dependent PGE2 synthesis ameliorates phagocytosis of fibrillar beta-amyloid peptide (1-42) via EP2 receptor in EMF-stimulated N9 microglial cells. J Neuroinflammation 13:296.

Hossain, M. Z., Peeling, J., Sutherland, G. R., Hertzberg, E. L., and Nagy, J. I. (1994). Ischemia-induced cellular redistribution of the astrocytic gap junctional protein connexin43 in rat brain. Brain Res. 652, 311-322. doi: 10.1016/0006-8993(94) 90242-9

Iyyathurai, J., D’hondt, C., Wang, N., De Bock, M., Himpens, B., Retamal, M. A., et al. (2013). Peptides and peptide-derived molecules targeting the intracellular domains of Cx43: gap junctions versus hemichannels. Neuropharmacology 75, 491-505. doi: 10.1016/j.neuropharm.2013.04.050

Ji, F. T., Liang, J. J., Miao, L. P., Wu, Q., and Cao, M. H. (2015). Propofol post-conditioning protects the blood brain barrier by decreasing matrix metalloproteinase- 9 and aquaporin-4 expression and improves the neurobehavioral outcome in a rat model of focal cerebral ischemia-reperfusion injury. Mol. Med. Rep. 12, 2049-2055. doi: 10.3892/mmr.2015.3585

Jiang, F., Yang, J., Zhang, L., Li, R., Zhuo, L., Sun, L., et al. (2014). Rosuvastatin reduces ischemia-reperfusion injury in patients with acute coronary syndrome treated with percutaneous coronary intervention. Clin. Cardiol. 37, 530-535.

Kalogeris, T., Baines, C. P., Krenz, M., and Korthuis, R. J. (2012). Cell biology of ischemia/reperfusion injury. Int. Rev. Cell Mol. Biol. 298, 229-317. doi: 10.1016/b978-0-12-394309-5.00006-7

Kurian, G. A., Rajagopal, R., Vedantham, S., and Rajesh, M. (2016). The role of oxidative stress in myocardial ischemia and reperfusion injury and remodeling: revisited. Oxid. Med. Cell. Longev. 2016:1656450.

Lee, C. H., Sapkota, A., Gaire, B. P., and Choi, J. W. (2020). NLRP3 inflammasome activation is involved in LPA1-mediated brain injury after transient focal cerebral ischemia. Int. J. Mol. Sci. 21:8595. doi: 10.3390/ijms21228595

Leithead, J. A., Armstrong, M. J., Corbett, C., Andrew, M., Kothari, C., Gunson, B. K., et al. (2013). Hepatic ischemia reperfusion injury is associated with acute kidney injury following donation after brain death liver transplantation. Transpl. Int. 26, 1116-1125. doi: 10.1111/tri.12175

Liu, X., Sun, K., Wang, H., and Dai, Y. (2016). Inhibition of autophagy by chloroquine enhances the antitumor efficacy of sorafenib in glioblastoma. Cell. Mol. Neurobiol. 36, 1197-1208. doi: 10.1007/s10571-015-0318-z

Longa, E. Z., Weinstein, P. R., Carlson, S., and Cummins, R. (1989). Reversible middle cerebral artery occlusion without craniectomy in rats. Stroke 20, 84-91. doi: 10.1161/01.str.20.1.84

Mussa, S., and Taggart, D. P. (2002). Myocardial and cerebral injury after off-pump coronary artery surgery. Circulation 105:e174. author reply e174,

Nakase, T., Söhl, G., Theis, M., Willecke, K., and Naus, C. C. (2004). Increased apoptosis and inflammation after focal brain ischemia in mice lacking connexin43 in astrocytes. Am. J. Pathol. 164, 2067-2075. doi: 10.1016/s00029440(10)63765-0

Naus, C. C., Bechberger, J. F., Caveney, S., and Wilson, J. X. (1991). Expression of gap junction genes in astrocytes and C6 glioma cells. Neurosci. Lett. 126, 33-36. doi: 10.1016/0304-3940(91)90364-y

Ohsumi, A., Nawashiro, H., Otani, N., Ooigawa, H., Toyooka, T., Yano, A., et al. (2006). Alteration of gap junction proteins (connexins) following lateral fluid percussion injury in rats. Acta Neurochir. Suppl. 96, 148-150. doi: 10.1007/3211-30714-1 33 
Pascual, O., Ben Achour, S., Rostaing, P., Triller, A., and Bessis, A. (2012). Microglia activation triggers astrocyte-mediated modulation of excitatory neurotransmission. Proc. Natl. Acad. Sci. U. S. A. 109, E197-E205.

Ponticelli, C. (2014). Ischaemia-reperfusion injury: a major protagonist in kidney transplantation. Nephrol. Dial. Transplant. 29, 1134-1140. doi: 10.1093/ndt/ gft488

Rash, J. E., Yasumura, T., and Dudek, F. E. (1998). Ultrastructure, histological distribution, and freeze-fracture immunocytochemistry of gap junctions in rat brain and spinal cord. Cell Biol. Int. 22, 731-749. doi: 10.1006/cbir.1998.0392

Schulz, R., Görge, P. M., Görbe, A., Ferdinandy, P., Lampe, P. D., and Leybaert, L. (2015). Connexin 43 is an emerging therapeutic target in ischemia/reperfusion injury, cardioprotection and neuroprotection. Pharmacol. Ther. 153, 90-106. doi: $10.1016 /$ j.pharmthera.2015.06.005

Song, S. H., Joo, H. T., Cho, H. W., Hwang, H. W., Lee, K. H., and Kim, D. K. (2011). Sex- and age-related changes in connexin 43 expression in normal rat bladder. Int. Neurourol. J. 15, 25-28. doi: 10.5213/inj.2011.15.1.25

Surinkaew, P., Sawaddiruk, P., Apaijai, N., Chattipakorn, N., and Chattipakorn, S. C. (2018). Role of microglia under cardiac and cerebral ischemia/reperfusion (I/R) injury. Metab. Brain Dis. 33, 1019-1030. doi: 10.1007/s11011-018-0232-4

Tao, T., Li, C. L., Yang, W. C., Zeng, X. Z., Song, C. Y., Yue, Z. Y., et al. (2016). Protective effects of propofol against whole cerebral ischemia/reperfusion injury in rats through the inhibition of the apoptosis-inducing factor pathway. Brain Res. 1644, 9-14. doi: 10.1016/j.brainres.2016.05.006

Taylor, R. A., and Sansing, L. H. (2013). Microglial responses after ischemic stroke and intracerebral hemorrhage. Clin. Dev. Immunol. 2013:746068.

Wang, L. Q., and Zhou, H. J. (2018). LncRNA MALAT1 promotes high glucose-induced inflammatory response of microglial cells via provoking MyD88/IRAK1/TRAF6 signaling. Sci. Rep. 8:8346.

Wentlandt, K., Samoilova, M., Carlen, P. L., and El Beheiry, H. (2006). General anesthetics inhibit gap junction communication in cultured organotypic hippocampal slices. Anesth. Analg. 102, 1692-1698. doi: 10.1213/01.ane. 0000202472.41103 .78
Xia, Q., Hu, Q., Wang, H., Yang, H., Gao, F., Ren, H., et al. (2015). Induction of COX-2-PGE2 synthesis by activation of the MAPK/ERK pathway contributes to neuronal death triggered by TDP-43-depleted microglia. Cell Death Dis. 6:e1702. doi: 10.1038/cddis.2015.69

Xu, S. Y., Wu, Y. M., Ji, Z., Gao, X. Y., and Pan, S. Y. (2012). A modified technique for culturing primary fetal rat cortical neurons. J. Biomed. Biotechnol. 2012:803930.

Yan, B. C., Ohk, T. G., Ahn, J. H., Park, J. H., Chen, B. H., Lee, J. C., et al. (2014). Differences in neuronal damage and gliosis in the hippocampus between young and adult gerbils induced by long duration of transient cerebral ischemia. J. Neurol. Sci. 337, 129-136. doi: 10.1016/j.jns.2013. 11.034

Yan, W., Chen, Z., Chen, J., and Chen, H. (2016). Isoflurane preconditioning protects rat brain from ischemia reperfusion injury via up-regulating the HIF-1alpha expression through Akt/mTOR/s6K activation. Cell. Mol. Biol. (Noisy-le-grand) 62, 38-44.

Zhou, X., Zoller, T., Krieglstein, K., and Spittau, B. (2015). TGFbetal inhibits IFNgamma-mediated microglia activation and protects $\mathrm{mDA}$ neurons from IFNgamma-driven neurotoxicity. J. Neurochem. 134, 125-134. doi: 10.1111/ jnc. 13111

Conflict of Interest: The authors declare that the research was conducted in the absence of any commercial or financial relationships that could be construed as a potential conflict of interest.

Copyright (C) 2021 Zhang, Wang, Xia, Tu, Sun, Jing, Chen and Zhao. This is an open-access article distributed under the terms of the Creative Commons Attribution License (CC BY). The use, distribution or reproduction in other forums is permitted, provided the original author(s) and the copyright owner(s) are credited and that the original publication in this journal is cited, in accordance with accepted academic practice. No use, distribution or reproduction is permitted which does not comply with these terms. 